\title{
Contractivity of Locally One-Dimensional Splitting Methods
}

\author{
J.G. Verwer
}

Mathematical Centre, Kruislaan 413, NL-1098 SJ Amsterdam, The Netherlands

\begin{abstract}
Summary. The aim of this paper is to study contractivity properties of two locally one-dimensional splitting methods for non-linear, multi-space dimensional parabolic partial differential equations. The term contractivity means that perturbations shall not propagate in the course of the time integration process. By relating the locally one-dimensional methods with contractive integration formulas for ordinary differential systems it can be shown that the splitting methods define contractive numerical solutions for a large class of non-linear parabolic problems without restrictions on the size of the time step.
\end{abstract}

Subject Classifications: AMS (MOS): 65L05; 65M05; 65M20; CR: 5.17.

\section{Introduction}

We restrict ourselves to the first initial boundary value problem for the single, $k$-space dimensional partial differential equation

$$
u_{t}=F u \equiv \sum_{i=1}^{k} F_{i}\left(t, x, u, \frac{\partial}{\partial x_{i}}\left(p_{i}(t, x) \frac{\partial u}{\partial x_{i}}\right)\right)
$$

where $t \in[0, \infty)$ and $x=\left(x_{1}, \ldots, x_{k}\right) \in \Omega, \Omega$ a bounded and connected region in $\mathbb{R}^{k}$ with boundary $\partial \Omega$. Throughout the paper the operator $F$ is assumed to be of elliptic type; then (1.1) is a parabolic equation. We also assume the existence of unique solutions $u$ for (1.1). By a solution of (1.1) we mean a continuous function $u$ having (at least) one continuous $t$-derivative and two continuous $x_{i}$-derivatives on $[0, \infty) \times(\Omega \cup \partial \Omega)$, which satisfies a given initial function and a given boundary function (see e.g. Friedman [7], Ch. 2.3).

More specifically, for the sake of our own analysis we require that the following conditions have been satisfied:

(1.2 a) $p_{i}$ and $\partial p_{i} / \partial x_{i}, i=1, \ldots, k$, are continuous and $p_{i}(t, x)>0$. 
(1.2b) for all reals $a, b$ the functions $F_{i} \equiv F_{i}(t, x, a, b), \partial F_{i} / \partial a, \partial F_{i} / \partial b$ are continuous, while $\partial F_{i}(t, x, a, b) / \partial a \leqq 0, \partial F_{i}(t, x, a, b) / \partial b>0$.

The conditions $p_{i}(t, x)>0, \partial F_{i}(t, x, a, b) / \partial b>0, i=1, \ldots, k$, mean that $F$ is elliptic and hence that (1.1) is a parabolic equation.

This paper deals with locally one-dimensional splitting methods. A method of this kind was first suggested by Yanenko (see Sect. 2.3 of his book [19]). If we follow the method of lines approach (semi-discretization in space), the essence of splitting methods can be described in a very compact way (cf. [8]).

Let the ordinary differential system

$$
\dot{y}=f(t, y), \quad f:[0, \infty) \times \mathbb{R}^{s} \rightarrow \mathbb{R}^{s},
$$

denote a semi-discrete version of (1.1) obtained after a finite difference or finite element (time continuous Galerkin) space-discretization. Suppose the vector function $f$ can be written as

$$
f(t, y)=\sum_{i=1}^{k} f_{i}(t, y), \quad f_{i}:[0, \infty) \times \mathbb{R}^{s} \rightarrow \mathbb{R}^{s},
$$

and in such a way that $f_{i}$ approximates the one-dimensional operator $F_{i}$. The combination of the space-discretization technique with the time integration formula

$$
\begin{aligned}
& y_{n+1}^{(0)}=y_{n}, \\
& y_{n+1}^{(i)}=y_{n+1}^{(i-1)}+\tau f_{i}\left(t_{n+1}^{(i)}, y_{n+1}^{(i)}\right), \quad i=1, \ldots, k, \\
& y_{n+1}=y_{n+1}^{(k)},
\end{aligned}
$$

then defines the most simple locally one-dimensional splitting method for nonlinear problems. Here $y_{n} \simeq y\left(t_{n}\right), \tau=t_{n+1}-t_{n}$ is the stepsize, $t_{n+1}^{(k)}=t_{n+1}, t_{n+1}^{(i)}=t_{n}$ $+c_{i} \tau$ for $i=1, \ldots, k-1$ where $0 \leqq c_{i} \leqq 1$.

Before we begin with our actual topic, contractivity, we wish to make a few remarks on the practical use of (1.5). The computation of the vectors $y_{n+1}^{(i)}$ from the implicit relations can be performed very cheaply using Newton iteration, since all Jacobian matrices $\partial f_{i} / \partial y$ can always be permuted to a banded form (one-dimensional structure). In fact, for each $i$, we can solve the implicit relations grid line per grid line in the $i$-th co-ordinate direction. This means that (1.5) is also very cheap with respect to the use of computer memory. Formula (1.5) requires only one array of storage, of length $s$, plus some additional work arrays, of much smaller length, for one-dimensional operations. The order of consistency of (1.5) is equal to one for all nonlinear functions $f$ satisfying the splitting relation (1.4). Hence this splitting formula should not be recommended for high accuracy calculations.

It is possible, however, to modify formula (1.5) in such a way that the order of consistency becomes two, whilst retaining the locally one-dimensional nature of the formula. We will present this second order formula in Sect. 4. Here we already observe that, after an appropriate scaling, the computational work per time step of the second order formula is exactly twice as large as that of formula (1.5). It is also worthwhile to observe that (1.5) can be shown to be 
contractive with respect to arbitrary vector norms in $\mathbb{R}^{s}$. This is impossible for the second order formula (cf. Spijker [13]), but it can be shown that this formula is contractive with respect to arbitrary inner product norms in $\mathbb{R}^{s}$.

\section{Dissipative Differential Systems and Contractive Integration Formulas}

The semi-discrete parabolic system (1.4) is a typical example of a stiff system. In recent years, since Dahlquist's paper on $G$-stability [4], there has been very promising developments towards a satisfactory stability and contractivity theory for numerical methods for stiff systems, in particular for non-linear ones. (See Spijker [14] for a recent review). The terms stability and contractivity mean here that in the course of the numerical calculations perturbations are not propagated unlimited and not propagated at all, respectively. It is natural to employ these developments for specific methods for timedependent partial differential equations, such as Yanenko's splitting method which we have written in the method of lines formulation (1.5). The fact that this integration formula bears a close resemblance to the well-known backward Euler formula means that it possesses optimal contractivity properties.

In this section we collect some known results on dissipativity and contractivity. Herewith we simply consider ordinary differential systems

$$
\begin{gathered}
\dot{y}=f(t, y), \quad f:[0, \infty) \times \mathbb{R}^{s} \rightarrow \mathbb{R}^{s}, \\
f(t, y)=\sum_{i=1}^{k} f_{i}(t, y), \quad f_{i}:[0, \infty) \times \mathbb{R}^{s} \rightarrow \mathbb{R}^{s},
\end{gathered}
$$

where $f_{i}, i=1, \ldots, k$, is continuous in $t$ and continuously differentiable in $y$ on the whole set $[0, \infty) \times \mathbb{R}^{s}$. By a solution of $(2.1)$ we mean a vector function $y(t)$ having its components in $C^{1}[0, \infty)$ and satisfying (2.1) on the whole interval $[0, \infty)$.

\subsection{Dissipative Differential Equations}

Throughout this paper $\|\cdot\|$ denotes a given vector norm on $\mathbb{R}^{s}$. The same symbol will be used for the norm of a real $s \times s$ matrix subordinate to the given vector norm. Given a square matrix $A$ and a vector norm $\|\cdot\|, \mu[A]$ will denote the logarithmic norm of $A$ with respect to the given vector norm, i.e. $\mu[A]=\lim _{\Delta \rightarrow 0_{+}}(\|I+\Delta A\|-1) / \Delta$. The logarithmic norm is a very useful tool in the analysis of non-linear differential equations (see e.g. Dahlquist $[4,5]$, Desoer and Haneda [6] and Ström [15]). The following result, quoted from Dahlquist [4], exemplifies this:

Theorem 1. Let $\|\cdot\|$ be a given norm. Let $v:[0, \infty) \rightarrow \mathbb{R}$ be a piecewise continuous function satisfying $\mu[\partial f(t, \zeta) / \partial y] \leqq v(t)$, all $(t, \zeta) \in[0, \infty) \times \mathbb{R}^{s}$. Then, for any two 
solutions $y$ and $\tilde{y}$ of (2.1) it holds that

$$
\left\|\tilde{y}\left(t_{2}\right)-y\left(t_{2}\right)\right\| \leqq \exp \left(\int_{t_{1}}^{t_{2}} v(\tau) d \tau\right)\left\|\tilde{y}\left(t_{1}\right)-y\left(t_{1}\right)\right\|
$$

for all $t_{1}, t_{2}$ satisfying $0 \leqq t_{1} \leqq t_{2}<\infty$.

Hence $\mu$ can be used to bound the difference between two solutions $y, \tilde{y}$ due to different initial values. The smoothness conditions on $f$ can be weakened somewhat (see Dahlquist [4], p. 12).

Of particular importance, for applications, is the case that $\mu$ is non-positive. Then (2.2) can be rewritten to (take $v(t) \equiv 0)$

$$
\left\|\tilde{y}\left(t_{2}\right)-y\left(t_{2}\right)\right\| \leqq\left\|\tilde{y}\left(t_{1}\right)-y\left(t_{1}\right)\right\|, \quad 0 \leqq t_{1} \leqq t_{2}<\infty .
$$

Hence exact solutions behave contractive with increasing $t$. In literature, differential equations with this property are called dissipative or monotone. We employ the following definition for the right hand side function:

Definition 2. Let $\|\cdot\|$ be a given norm. Let the function $f(t, y)$, where $f:[0, \infty)$ $\times \mathbb{R}^{s} \rightarrow \mathbb{R}^{s}$, be continuous in $t$ and continuously differentiable in $y$ on the whole set $[0, \infty) \times \mathbb{R}^{s}$. Then $f$ is called dissipative, w.r.t. the given norm, if $\mu[\partial f(t, \zeta) / \partial y] \leqq 0$, all $(t, \zeta) \in[0, \infty) \times \mathbb{R}^{s}$.

\subsection{Contractive Integration Formulas}

Let the implicit relation

$$
y_{n+1}=y_{n}+\tau \Phi\left[\tau, y_{n}, y_{n+1}\right], \quad n \geqq 0, y_{0} \text { given, } \tau=t_{n+1}-t_{n},
$$

represent an integration formula for the numerical solution of the differential system $\dot{y}=f(t, y)$. If this system is dissipative, i.e. if $f$ is dissipative, it makes sense to think about contractivity for the numerical solution too:

Definition 3. Let $f$ be dissipative w.r.t. a given norm $\|$.$\| . Integration formula$ (2.4) is called contractive for this $f$, w.r.t. the same norm, if

$$
\left\|\tilde{y}_{n+1}-y_{n+1}\right\| \leqq \kappa_{n+1}\left\|\tilde{y}_{n}-y_{n}\right\|, \quad \kappa_{n+1} \leqq 1 \quad \text { for all } \tau>0,
$$

for any two sequences $\left\{\tilde{y}_{n}\right\},\left\{y_{n}\right\}$ defined by this formula.

Note that we have defined contractivity as an unconditional property with respect to $\tau$, i.e. inequality (2.5) should not lead to a restriction on the step size. This is of importance when dealing with stiff, and hence with semi-discrete parabolic equations. When writing down inequality (2.5) we have assumed that (2.4) is a meaningful relation in the sense that, for given $\tau$ and $y_{n}$, the solution $y_{n+1}$ exists. Note that when this is true, contractivity implies uniqueness. The significance of the notion of contractivity for the numerical practice is pointed out below. 


\subsection{The Propagation of Errors for Contractive Formulas}

Unconditionally contractive integration methods are implicit. Consequently, in actual computation one has to implement such a method in combination with some sort of iteration process, usually of Newton type, to solve for the implicit relations. One then may question whether the resulting implementation, considered as a new method on its own, shares the contractivity properties of the underlying integration formula. As pointed out in $[9,12,16,18]$, the answer to this question will always be negative, unless the class of differential equations is narrowed (see Hundsdorfer [9]). Alternatively, one may question how errors made in solving the implicit relations propagate from step to step. At the same time the question arises how the local discretization error of the integration formula propagates. The answer to both questions is given in the following familiar theorem, the proof of which is standard.

Theorem 4. Let $\|\cdot\|$ be a given norm and $f(t, y)$ a dissipative function w.r.t. this norm. Let (2.4) be contractive for this $f$ in the same norm and be applied to $\dot{y}=f(t, y), 0 \leqq t<\infty, y(0)=y_{0}$. Let $y(t)$ denote the exact solution of this initial value problem. We have

(i) Let, for $n \geqq 0, l_{n+1}$ be the local discretization error of (2.4) with respect to $y(t)$, i.e. $l_{n+1}=y\left(t_{n+1}\right)-\hat{y}_{n+1}, \hat{y}_{n+1}=y\left(t_{n}\right)+\tau \Phi\left[\tau, y\left(t_{n}\right), \hat{y}_{n+1}\right]$. Then for all $\tau>0$ it holds that

$$
\left\|y\left(t_{n+1}\right)-y_{n+1}\right\| \leqq \kappa_{n+1}\left\|y\left(t_{n}\right)-y_{n}\right\|+\rho_{n+1}, \quad \kappa_{n+1} \leqq 1,
$$

where $\kappa_{n+1}$ is the same constant as introduced in inequality (2.5) and $\rho_{n+1}$ $=\left\|l_{n+1}\right\|$.

(ii) Suppose that we compute a sequence $\left\{v_{n}\right\}$, instead of $\left\{y_{n}\right\}$, such that

$$
\left\|v_{n+1}-\tilde{y}_{n+1}\right\| \leqq \varepsilon_{n+1}, \quad \tilde{y}_{n+1}=v_{n}+\tau \Phi\left[\tau, v_{n}, \tilde{y}_{n+1}\right] .
$$

Then, again for all $\tau>0$, it holds that

$$
\left\|y_{n+1}-v_{n+1}\right\| \leqq \kappa_{n+1}\left\|y_{n}-v_{n}\right\|+\varepsilon_{n+1} .
$$

(iii) Let, for $n \geqq 0$ and given $\tau$-values, $\kappa_{n+1} \leqq k \leqq 1, \varepsilon_{n+1} \leqq \varepsilon, \rho_{n+1} \leqq \rho$. Then the true global error $y\left(t_{n+1}\right)-v_{n+1}$ satisfies the inequality

provided $v_{0}=y_{0}$.

$$
\left\|y\left(t_{n+1}\right)-v_{n+1}\right\| \leqq(\rho+\varepsilon) \sum_{i=0}^{n} \kappa^{i}
$$

Inequalities (2.6), (2.8) show that there is no propagation of local errors, since $\kappa_{n+1} \leqq 1$. Note that nothing has been said about the way $\left\{v_{n}\right\}$ has been computed. Thus, for example, $\varepsilon_{n+1}$ may represent iteration errors, but also rounding errors. Inequality (2.9) reflects the importance of unconditional contractivity. It shows that when a contractive formula is implemented on a 
computer the true global error after $n$ steps, irrespective of the value of $\tau$, is always bounded by $n$ times the maximum of the total local errors, i.e. local discretization errors plus local computational errors.

\section{Contractivity of Yanenko's Time Integration Formula}

In this section we present a theorem on the contractivity of the specific integration formula (1.5) for differential systems (2.1).

Lemma 5. Let $\|\cdot\|$ be a given norm. Let the functions $f_{i}(t, y), i=1, \ldots, k$, satisfy the hypotheses made for (2.1). Then, for all $(t, \zeta) \in[0, \infty) \times \mathbb{R}^{s}$, it follows that $\mu[\partial f(t, \zeta) / \partial y] \leqq \mu\left[\partial f_{1}(t, \zeta) / \partial y\right]+\ldots+\mu\left[\partial f_{k}(t, \zeta) / \partial y\right]$.

Proof. For any norm and all square matrices $A, B$ one has $\mu[A+B] \leqq \mu[A]$ $+\mu[B]$.

Hence, if all functions $f_{i}$ are dissipative, the sum function $f$ shares this property. The reverse need not to be true.

Lemma 6. Let $\|\cdot\|$ be a given norm. Suppose that $f(t, y)$ is dissipative w.r.t. to this norm. Then Backward Euler is contractive for this function $f$.

Proof. This nice property of Backward Euler is already known for some time (see e.g. Desoer and Haneda [6]). Because this property plays a key role in our paper we present a short proof for it. In fact, a slightly more general result can be proven.

Let $\|\cdot\|$ be an arbitrary norm. Let $f(t, y)$ satisfy the hypotheses made for (2.1). Let $v$ have the same meaning as in Theorem 1. Let $\left\{v_{n}\right\},\left\{\tilde{v}_{n}\right\}$ denote two sequences of numerical solutions for $\dot{y}=f(t, y)$ defined by Backward Euler, i.e.

$$
v_{n+1}=v_{n}+\tau f\left(t_{n+1}, v_{n+1}\right), \quad \tilde{v}_{n+1}=\tilde{v}_{n}+\tau f\left(t_{n+1}, \tilde{v}_{n+1}\right) .
$$

Subtraction and application of the mean value theorem leads to

$$
\begin{gathered}
(I-\tau M)\left(\tilde{v}_{n+1}-v_{n+1}\right)=\tilde{v}_{n}-v_{n}, \\
M=\int_{0}^{1} \partial f\left(t_{n+1}, \theta \tilde{v}_{n+1}+(1-\theta) v_{n+1}\right) / \partial y d \theta .
\end{gathered}
$$

From properties 5), 6), 10) from Desoer and Haneda [6], p. 480, it follows that

$$
\left\|\tilde{v}_{n}-v_{n}\right\| \geqq(1-\tau \mu[M])\left\|\tilde{v}_{n+1}-v_{n+1}\right\| .
$$

Further, using a result from Dahlquist [4], p. 11, one finds $\mu[M] \leqq v\left(t_{n+1}\right)$, so that

$$
\left\|\tilde{v}_{n+1}-v_{n+1}\right\| \leqq \kappa_{n+1}\left\|\tilde{v}_{n}-v_{n}\right\| \quad \text { if } \tau v\left(t_{n+1}\right)<1,
$$

where $\kappa_{n+1}=\left(1-\tau \nu\left(t_{n+1}\right)\right)^{-1}$. If $f$ is dissipative, $v$ can be chosen such that $v(t) \leqq 0, t \geqq 0$. 
Theorem 7. Let $\|\cdot\|$ be some given norm. Suppose that $f_{i}(t, y), i=1, \ldots, k$, is dissipative w.r.t. this norm. Then Yanenko's time integration formula (1.5) is contractive for the sum function $f$.

Proof. Let $\|\cdot\|$ be an arbitrary norm. Let $f_{i}(t, y), i=1, \ldots, k$, satisfy the hypotheses made for (2.1). First we observe that if all $f_{i}$ are dissipative w.r.t. $\|\cdot\|$, then $f$ is also dissipative w.r.t. this norm as shown by Lemma 5 . Thus it is appropriate to investigate the contractivity of formula (1.5) when all functions $f_{i}$ are dissipative.

One integration step with (1.5) may be considered as $k$ consecutive steps with the backward Euler scheme, but each time with a different right hand side function. This means that the results of Lemma 6 can be carried over immediately since each $f_{i}$ has been assumed to be dissipative. More precisely, let $v_{i}:[0, \infty) \rightarrow \mathbb{R}$ be a piecewise continuous function satisfying $\mu\left[\partial f_{i}(t, \zeta) / \partial y\right] \leqq v_{i}(t)$, all $(t, \zeta) \in[0, \infty) \times \mathbb{R}^{s}$, for $i=1, \ldots, k$. For any two exact solutions of $\dot{y}=f(t, y)$, inequality (2.2) is then valid where $v(t)=v_{1}(t)+\ldots+v_{k}(t)$. For any two numerical solutions $\left\{y_{n}\right\},\left\{\tilde{y}_{n}\right\}$ of $\dot{y}=f(t, y)$, defined by formula (1.5), one has (see (3.4))

$\left\|\tilde{y}_{n+1}-y_{n+1}\right\| \leqq \prod_{i=1}^{k} \frac{1}{1-\tau v_{i}\left(t_{n+1}^{(i)}\right)}\left\|\tilde{y}_{n}-y_{n}\right\| \quad$ if $\quad \tau v_{i}\left(t_{n+1}^{(i)}\right)<1, \quad i=1, \ldots, k$.

If all $f_{i}$ are dissipative, all $v_{i}$ can be chosen to satisfy $v_{i}(t) \leqq 0, t \geqq 0$.

It goes without saying that the results of Theorem 4 on the propagation of errors can be applied to our specific integration formula (1.5).

\section{Contractivity of a Second Order Locally One-Dimensional Time Integration Formula}

A disadvantage of integration formula (1.5) is its low order of consistency. In this section we will discuss a second order one.

\subsection{A Second Order Locally One-Dimensional Integration Formula}

It is not difficult to prove that the order of consistency of formula (4.1) equals two for all, sufficiently smooth, non-linear functions $f(t, y)$ satisfying the linear splitting relation (1.4):

$$
\begin{aligned}
y_{n+\frac{1}{2}}^{(0)} & =y_{n}, \\
y_{n+\frac{1}{2}}^{(i)} & =y_{n+\frac{1}{2}}^{(i-1)}+\frac{1}{2} \tau f_{i}\left(t_{n}+\frac{1}{4} \tau, \frac{1}{2} y_{n+\frac{1}{2}}^{(i-1)}+\frac{1}{2} y_{n+\frac{1}{2}}^{(i)}\right), \quad i=1, \ldots, k, \\
y_{n+\frac{1}{2}} & =y_{n+\frac{1}{2}}^{(k)} \\
y_{n+1}^{(k)} & =y_{n+\frac{1}{2}}, \\
y_{n+1}^{(i-1)} & =y_{n+1}^{(i)}+\frac{1}{2} \tau f_{i}\left(t_{n}+\frac{3}{4} \tau, \frac{1}{2} y_{n+1}^{(i-1)}+\frac{1}{2} y_{n+1}^{(i)}\right), \quad i=k, \ldots, 1, \\
y_{n+1} & =y_{n+1}^{(0)} .
\end{aligned}
$$


Here $y_{n} \simeq y\left(t_{n}\right), \tau=t_{n+1}-t_{n}$ is the stepsize for the computation $y_{n} \rightarrow y_{n+1}$. Formulas (4.1a), (4.1b) are both first order consistent in the stepsize $\tau / 2$. Apart from the order of the functions $f_{i},(4.1 \mathrm{a})$ and $(4.1 \mathrm{~b})$ are identical. In fact, the symmetry in the order of the functions $f_{i}$ is necessary for obtaining order of consistency two. Note that formula (4.1) shares all computational advantages of formula (1.5) which we mentioned in the introduction. Per complete time step $t_{n} \rightarrow t_{n+1},(4.1)$ requires twice the computational work of (1.5).

For constant coefficient linear problems

$$
\dot{y}=J y=\sum_{i=1}^{k} J_{i} y,
$$

(4.1 a), as well as (4.1b), is second order consistent in the stepsize $\tau / 2$, if and only if all matrices $J_{i}$ commute with each other (Yanenko [19], p. 23). To maintain second order consistency for non-commuting matrices, Marchuk [11] suggested to reverse the order of the matrices $J_{i}$ on every other (half) time step. For the general non-linear case we recommend the specific splitting scheme (4.1), since this scheme can be shown to be contractive in arbitrary inner product norms.

\subsection{Contractivity of the Second Order Formula}

Like formula (1.5) is related to Backward Euler, formulas (4.1 a) and (4.1 b) stem from the implicit midpoint or one-leg trapezoidal rule

$$
v_{n+1}=v_{n}+\tau f\left(t_{n}+\frac{1}{2} \tau, \frac{1}{2} v_{n}+\frac{1}{2} v_{n+1}\right)
$$

for the numerical integration of $\dot{y}=f(t, y)$. This enables us to prove contractivity for the second order splitting formula (4.1). We shall follow the same method of proof as in Sect. 3.

Lemma 8. Let $\|\cdot\|$ be some given inner product norm. Suppose that the function $f(t, y)$ is dissipative w.r.t. this norm. Then the implicit midpoint rule (4.3) is contractive for this function $f$.

Proof. This nice property of (4.3) is also known for some time (see Dahlquist [5], Burrage and Butcher [1], Crouzeix [3]). We shall present a short proof which is based on the logarithmic norm.

Let $\langle.,$.$\rangle be a given inner product in \mathbb{R}^{s}$ and define $\|v\|=\langle v, v\rangle^{\frac{1}{2}}, v \in \mathbb{R}^{s}$. Let $f(t, y)$ satisfy the hypotheses made for system (2.1). Let $v$ have the same meaning as in Theorem 1. Let $\left\{v_{n}\right\},\left\{\tilde{v}_{n}\right\}$ denote two sequences of numerical solutions defined by formula (4.3). Then, subtraction and application of the mean value theorem leads to

$$
\begin{aligned}
& \left(I-\frac{1}{2} \tau M\right) e_{n+1}=\left(I+\frac{1}{2} \tau M\right) e_{n}, \\
& M=\int_{0}^{1} \partial f\left(t_{n}+\frac{1}{2} \tau, \theta\left[\frac{1}{2} \tilde{v}_{n}+\frac{1}{2} \tilde{v}_{n+1}\right]+(1-\theta)\left[\frac{1}{2} v_{n}+\frac{1}{2} v_{n+1}\right]\right) / \partial y d \theta,
\end{aligned}
$$


where $e_{n}=\tilde{v}_{n}-v_{n}$. Following Ström [15] and Kellogg [10], we now write $e_{n+1}-e_{n}=\frac{1}{2} \tau M\left(e_{n+1}+e_{n}\right)$ and form the inner product with $e_{n}+e_{n+1}$ which results into $\left\|e_{n+1}\right\|^{2}-\left\|e_{n}\right\|^{2}=\left\langle e_{n}+e_{n+1}, \frac{1}{2} \tau M\left(e_{n}+e_{n+1}\right)\right\rangle$. Then from $\mu[M]$ $=\sup \{\langle v, M v\rangle:\|v\|=1\}$ and $\mu[M] \leqq v\left(t_{n}+\frac{1}{2} \tau\right)$, we find

$$
\left\|e_{n+1}\right\|^{2}-\left\|e_{n}\right\|^{2} \leqq \frac{1}{2} \tau \nu\left(t_{n}+\frac{1}{2} \tau\right)\left\|e_{n}+e_{n+1}\right\|^{2} .
$$

If $f$ is dissipative, a function $v$ exists satisfying $v(t) \leqq 0$, which implies $\left\|e_{n+1}\right\| \leqq \kappa_{n+1}\left\|e_{n}\right\|, \kappa_{n+1} \leqq 1$, i.e. contractivity.

Theorem 9. Let $\|\cdot\|$ be some given inner product norm. Suppose that $f_{i}(t, y)$, $i=1, \ldots, k$, is dissipative w.r.t. this norm. Then the second order locally onedimensional integration formula (4.1) is contractive for the sum function $f$.

Proof. By making use of Lemma 8 the proof can be given in a completely analogous way as the proof of Theorem 7 .

Observe that for the implicit midpoint rule the upperbound $v(t)$ of the logarithmic norm cannot be explicitly substituted into the damping parameter $\kappa_{n+1}$ like for Backward Euler (see (3.4)). This can easily be understood from the behaviour of the midpoint rule for the scalar test equation $\dot{y}=\delta y$. However, if $v(t)<0, t \geqq 0$, inequality (4.5) reveals that $\kappa_{n+1}<1$.

\section{A Dissipative Semi-Discrete Parabolic Equation}

For two reasons the results presented in Sects. 3 and 4 are very general. Firstly, the ordinary differential system considered, viz. (2.1), is not necessarily related to a prabolic Eq. (1.1) with Dirichlet boundary conditions. More general boundary conditions, even in combination with other equations, e.g., hyperbolic equations or systems of equations, may also give rise to ordinary differential systems of the form (2.1). Secondly, as we did not select one specific norm beforehand, in particular in Sect. 3, it is still possible to choose a norm which is appropriate for a given application.

This section deals with an application for Theorem 7 on the first order splitting formula. We will show that if the first initial boundary value problem for Eq. (1.1) is semi-discretized with symmetrical 3-point finite differences, then the resulting system of ordinary differential equations is always dissipative in the maximum norm.

\subsection{Description of the Semi-Discrete Problem}

Consider Eq. (1.1) and assume that conditions (1.2) have been satisfied. Further assume that the domain $\Omega \cup \partial \Omega$ has been covered by an orthogonal $k$-dimensional grid, $\Omega_{h} \cup \partial \Omega_{h}$ say. The grid need not to be uniform, but we assume that all grid points from $\partial \Omega_{h}$ are located on $\partial \Omega$. Then, for each $x \in \Omega_{h}$, we can replace the one-dimensional expression $\left(p_{i}(t, x) u_{x_{i}}\right)_{x_{i}}$ by the belonging 3-point finite difference expressions in the $i$-th co-ordinate direction. 
Let $x \in \Omega_{h}$. Let $x_{i \pm} \in \Omega_{h} \cup \partial \Omega_{h}$ denote the nearest neighbouring grid points in the $i$-th co-ordinate direction. The component of $f_{i}$ corresponding to the grid point $x$ can then be represented as

$$
F_{i}\left(t, x, U(t, x),\left(\frac{2}{x_{i_{+}}-x_{i_{-}}}\right)\left(s_{-} U\left(t, x_{i-}\right)-\left(s_{-}+s_{+}\right) U(t, x)+s_{+} U\left(t, x_{i+}\right)\right)\right),
$$

where $s_{ \pm}$is an abbreviation for $p_{i}\left(t, \frac{1}{2}\left(x+x_{i \pm}\right)\right) /\left|x-x_{i \pm}\right|$ and $U(t, x)$ stands for the semi-discrete approximation to $u(t, x)$. In case $x_{i \pm} \in \partial \Omega_{h}$, known solution values must be substituted for $U\left(t, x_{i \pm}\right)$. If we assume a natural numbering for all grid points $x \in \Omega_{h}$, the approximations $U(t, x)$ assemble the vector $y(t)$. We thus arrive at the semi-discrete parabolic equation (s grid points in $\Omega_{h}$ )

$$
\dot{y}=f(t, y)=\sum_{i=1}^{k} f_{i}(t, y), \quad f_{i}:[0, \infty) \times \mathbb{R}^{s} \rightarrow \mathbb{R}^{s} .
$$

Note that $f_{i}(t, y), i=1, \ldots, k$, is continuous in $t$ and continuously differentiable in $y$ on $[0, \infty) \times \mathbb{R}^{s}$.

For all grid functions $\zeta \in \mathbb{R}^{s}$ the Jacobian matrices $\partial f_{i}(t, \zeta) / \partial y$ take the form

$$
J_{i}=D_{1}+D_{2} D_{3} S,
$$

where $D_{1}, D_{2}, D_{3}$ are $s \times s$ diagonal matrices and $S$ is a symmetric $s \times s$ matrix having three non-zero diagonals. $D_{1}$ contains, for all grid points, the derivative of $F_{i}$ w.r.t. its third argument. By assumption (see (1.2)), $D_{1}$ is non-positive definite. In a similar way, $D_{2}$ contains the derivatives of $F_{i}$ w.r.t. its fourth argument. By assumption, $D_{2}$ is positive definite. $D_{3}$ contains all numbers $2 /\left(x_{i+}-x_{i-}\right)$ and hence is also positive definite. Finally, the entries of $S$, at most three in each row, are just the numbers $s_{-},-\left(s_{-}+s_{+}\right), s_{+}$. By assumption, $s_{-}$and $s_{+}$are always positive.

In fact $S$, and hence $J_{i}$, can be written as the direct sum of irreducible matrices of a much smaller dimension. Namely, for each grid line in the $i$-th co-ordinate direction we have one such irreducible matrix. By an appropriate reordering, these matrices can always be brought into a tridiagonal form (see e.g. Varga [17]).

\subsection{Dissipativity Measured in the Maximum Norm}

According to Theorem 7, any vector norm in $\mathbb{R}^{s}$ can be used for testing the condition of dissipativity for the vector functions $f_{i}$ defined in the preceding subsection. Recall that if all $f_{i}$ are dissipative, then the sum function $f$ is dissipative, while the integration formula (1.5) is contractive. Let us consider the maximum norm $\|v\|_{\infty}=\max _{j}\left|v_{j}\right|, v \in \mathbb{R}^{s}$.

Theorem 10. All vector functions $f_{i}$ of the semi-discrete parabolic equation (5.2) derived in Sect. 5.1 are dissipative in the maximum norm. 
Proof. $\mu_{\infty}[A], A$ being a real $s \times s$ matrix $\left(a_{j k}\right)$, is given by

$$
\mu_{\infty}[A]=\max _{j}\left(a_{j j}+\sum_{k \neq j}\left|a_{j k}\right|\right) .
$$

Hence, if all diagonal entries of $A$ are negative and $A$ is diagonally dominant, then $\mu_{\infty}[A] \leqq 0$. Consider the Jacobian matrix (5.3). All diagonal entries of $S$ are negative and $S$ is obviously diagonally dominant. The same is true for $J_{i}$, since $D_{1}$ is non-positive definite and $D_{2} D_{3}$ is positive definite. This implies that $\mu_{\infty}\left[J_{i}\right] \leqq 0$.

The proof shows that the maximum norm is easy to use for testing the condition of dissipativity. Regarding the fact that is is advantageous to find a negative logarithmic norm, since then the $\kappa_{n+1}$ of Definition 3 satisfy $\kappa_{n+1}<1$ (cf. (3.5), (3.4)), one might wonder however, whether for the present application the maximum norm is logarithmically optimal (cf. Ström [15]):

Definition 11. A norm is called logarithmically optimal w.r.t. a matrix $A$, if $\mu[A]=\alpha[A]$, where $\alpha[A]$ denotes the maximal real part of the eigenvalues of A.

The meaning of this definition stems from the inequality $\alpha[A] \leqq \mu[A]$, which is valid for all norms. Hence a better value than $\alpha[A]$ cannot be obtained.

From the definitions of $\mu_{\infty}$ and $J_{i}$, it is immediate that

$$
\mu_{\infty}\left[J_{i}\right]=\mu_{\infty}\left[D_{1}\right]=\text { largest element of } D_{1} .
$$

Hence the matrix $S$ does not contribute at all to $\mu_{\infty}\left[J_{i}\right]$. Further, there always exists an $s \times s$ permutation matrix $P$ such that $P S P^{T}$ is the direct sum of irreducibly diagonally dominant matrices of tridiagonal form, which implies that $\alpha[S]$, and hence $\alpha\left[J_{i}\right]$, is negative (see Varga [17]).

We thus see that for the present application the maximum norm is not logarithmically optimal. It remains an advantage of course that with the maximum norm one can easily show dissipativity for the whole class of non-linear problems (5.2).

One should realize that $\alpha[S]<0$ thanks to the Dirichlet boundary conditions assumed for (1.1). If we also admit Neumann conditions, we might have to deal with matrices $S=\left(s_{j k}\right)$, where, for all $j$,

$$
\sum_{k} s_{j k}=0, \quad s_{j j} \leqq 0, \quad s_{j k} \geqq 0, \quad k \neq j .
$$

Such a matrix does have a zero eigenvalue (see Varga [17], p. 24), while the method of proof of Theorem 10 goes through. Hence, when Neumann conditions are assumed, for a lot of problems $\mu_{\infty}$ might even be shown to be logarithmically optimal.

\section{Final Remarks}

We have discussed contractivity properties of two locally one-dimensional splitting methods for an interesting class of nonlinear, $k$-space dimensional 
parabolic differential equations. Since contractivity is a property of the timeintegration we concentrated our analysis around the space-discretized problem, i.e. we followed the method of lines approach. We thus were able to make fruitful use of some nice results from the numerical analysis of stiff ordinary differential systems and showed that the locally one-dimensional splitting methods possess ideal contractivity properties under rather general circumstances. This indicates that other classes of partial differential equations, such as systems of equations, hyperbolic and mixed parabolic-hyperbolic equations, can be treated likewise.

In the preceding section we embarked upon the problem of measuring dissipativity. By way of application we discussed a semi-discrete version of the general parabolic Eq. (1.1) when provided with Dirichlet boundary conditions. It was not difficult to prove that this general semi-discrete problem is dissipative in the maximum norm. If we only impose Dirichlet conditions, however, the maximum norm cannot be logarithmically optimal.

We wish to emphasize that the problem of measuring dissipativity deserves much more attention than given in Sect. 5 (see also Ström [15]). Because our system of ordinary differential equations originates from a partial differential equation one could think over to deduce dissipativity of the ordinary differential system from dissipativity properties of the partial differential equation itself (cf. Ciarlet et al. [2]). This might be helpful in selecting a norm, e.g. an inner product norm, which is (almost) logarithmically optimal and suited for the splitting method under consideration.

Acknowledgement. The author wishes to acknowledge his colleague Dr. K. Dekker for his careful reading of the manuscript.

\section{References}

1. Burrage, K., Butcher, J.C.: Stability criteria for implicit Runge-Kutta methods. SIAM J. Numer. Anal. 16, 46-57 (1979)

2. Ciarlet, P.G., Schultz, M.H., Varga, R.S.: Numerical methods of high order accuracy for nonlinear boundary value problems V: Monotone Operators. Numer. Math. 13, 51-77 (1969)

3. Crouzeix, M.: Sur la B-stabilité des méthodes de Runge-Kutta. Numer. Math. 32, 75-82 (1979)

4. Dahlquist, G.: Stability and error bounds in the numerical integration of ordinary differential equations. Trans. Roy. Inst. Tech. No. 130, Stockholm 1959

5. Dahlquist, G.: Error analysis for a class of methods for stiff non-linear initial value problems. In: Lecture Notes in Mathematics 506. Berlin, Heidelberg, New York: Springer 1976

6. Desoer, C.A., Haneda, H.: The measure of a matrix as a tool to analyze computer algorithms for circuit analysis. IEEE Trans. on Circuit Theory 19, 480-486 (1972)

7. Friedman, A.: Partial differential equations of parabolic type. Englewood Cliffs, N.J.: Prentice Hall Inc. 1964

8. Houwen, P.J. van der, Verwer, J.G.: One-step splitting methods for semi-discrete parabolic equations, Computing 22, 291-309 (1979)

9. Hundsdorfer, W.H.: Non-linear stability analysis for a simple Rosenbrock method. Report 8131, University of Leiden 1981

10. Kellogg, R.B.: An alternating direction method for operator equations. J. Soc. Ind. Appl. Math. 12, 848-854 (1964)

11. Marchuk, G.I.: On the theory of the splitting-up method. In: Numerical Solution of Partial Differential Equations - II (B. Hubbard, ed.). New York, London: Academic Press 1971 
12. Sandberg, I.W., Shichman, H.: Numerical integration of systems of stiff non-linear differential equations. Bell System Tech. J. 47, 511-527 (1968)

13. Spijker, M.N.: Contractivity of Runge-Kutta methods. In: Numerical methods for solving stiff initial value problems, Bericht Nr. 9 der Institut für Geometrie und Praktische Mathematik der RWTH Aachen (G. Dahlquist, R. Jeltsch, eds.) 1981

14. Spijker, M.N.: Stability in the numerical solution of stiff initial value problems. Nieuw Arch. Wisk. 30, 235-246 (1982)

15. Ström, T.: On logarithmic norms. SIAM J. Numer. Anal. 12, 741-753 (1975)

16. Vanselow, R.: Stabilität und Fehleruntersuchungen bei numerischen Verfahren zur Lösung steifer nichtlinearer Anfangswertprobleme. Diplomarbeit Sektion Mathematik TU-Dresden 1979

17. Varga, R.S.: Matrix iterative analysis. Englewood Cliffs N.J.: Prentice Hall Inc. 1962

18. Verwer, J.G.: On the practical value of the notion of BN-stability. BIT 21, 355-361 (1981)

19. Yanenko, N.N.: The method of fractional steps. Berlin, Heidelberg, New York: Springer 1971

Received March 8, 1982 / December 18, 1983 